\title{
Triple-wavelength filter based on the nanoplasmonic metal-insulator-metal waveguides
}

Cao Dung Truong ( $\nabla$ dung.truongcao@hust.edu.vn )

Electronics and Telecommunicatons

\section{Tai Nguyen Van}

Posts and Telecommunications Institute of Technology

\section{Minh Tuan Trinh}

University of South Florida

Hoang Chu Manh

Hanoi University of Technology

\section{Hung Nguyen Tan}

The University of Danang-Danang University of Science and Technology

\section{Bac Dang Hoai}

Posts and Telecommunications Institute of Technology

\section{Original Research}

Keywords: surface plasmon polaritons (SPPs), metal-insulator-metal (MIM), Fabry-Perot nanocavity, wavelength filter, eigen mode expansion (EME) simulation

Posted Date: February 1st, 2021

DOI: https://doi.org/10.21203/rs.3.rs-177604/v1

License: (1) (i) This work is licensed under a Creative Commons Attribution 4.0 International License.

Read Full License

Version of Record: A version of this preprint was published at Optical and Quantum Electronics on April 21st, 2021. See the published version at https://doi.org/10.1007/s11082-021-02902-2. 


\title{
Triple-wavelength filter based on the nanoplasmonic metal-insulator-metal waveguides
}

\author{
Cao Dung Truong ${ }^{1 *}$, Tai Nguyen Van ${ }^{1}$, Minh Tuan Trinh ${ }^{2}$, \\ Hoang Chu Manh ${ }^{3}$, Hung Nguyen Tan ${ }^{4}$, and Bac Dang Hoai ${ }^{1}$ \\ ${ }^{1}$ Posts and Telecomunications Institute of Technology, Vietnam \\ ${ }^{2}$ Michigan University, USA \\ ${ }^{3}$ International Training Institute for Materials Science- Hanoi University of Science and Technology, Vietnam \\ ${ }^{4}$ The University of Danang- Danang University of Science and Technology \\ *Corresponding author: dungtc@ptit.edu.vn
}

\begin{abstract}
In this paper, we present a proposal for compact photonic wavelength filtering and 3-dB wavelength splitting device based on nanoplasmonic metal-insulator-metal structure. The working performance of the device has been accurately simulated using the temporal coupled-mode theory. We use a numerical simulation method of eigenmode expansion propagation for the overall design process. We show that the transmission efficiency of the drop filter can be significantly enhanced by applying specifically optimization of nanostub waveguide. The proposed structure has potential applications in highly efficient ultracompact integration circuits as well as in optical communication systems at nanoscale.
\end{abstract}

Keywords - surface plasmon polaritons (SPPs), metalinsulator-metal (MIM), Fabry-Perot nanocavity, wavelength filter, eigen mode expansion (EME) simulation.

\section{INTRODUCTION}

Recently, surface plasmon polaritons (SPPs) have proved the considerable potential to confine light in a nanoscale beyond the so-called diffraction limit that was unable to overcome by silicon-based photonic waveguides[1]-[3]. SPPs are regarded as a promising method for constructing highly integrated photonic circuits [4]-[6], for instance, long-range SPPs can be realized in a plasmonic hybrid silicon waveguide [7],[8]. Among SSP structures, plasmon-slot based waveguides using metal-insulator-metal (MIM) structures have been realized in both long-range propagation and sub-wavelength SSP confinement for photonic integrated circuits[9],[10]. Moreover, MIM waveguides are widely applied in designing nanoscale integrated devices because of high localization and small bending loss with a relatively easy fabrication thanks to the current advanced manufacturing technologies. These plasmonic nanostructures were applied to design variety of devices, for instance, switches [5], [11], [12], sub-wavelength imaging systems [13], biosensors [14],[15]. Furthermore, broadband MIM waveguides with low side lobes were proposed as an alternative to create Bragg gratings for coupling with optical fibers in telecommunication systems [16]. Metallic plasmonic slits used for the design of nanoscale devices with near-field applications have been demonstrated in the fact [17] because MIM waveguides can trap light with an acceptable length for SPP propagation [10]. Based on the MIM waveguide architecture, recently, several wavelength-selective devices have been proposed and investigated, e.g. plasmonic toothshaped filters [18],[19], and nano-disk shaped resonators [20].
In various kinds of MIM waveguides, optical resonators using nanotubes are important elements of plasmonic wavelengthmultiplexing structures due to their symmetry, simplicity, and simple of fabrication [21].

Plasmonic wavelength demultiplexers allowing to filter specific wavelengths in channels play an important role in all-optical wavelength processing nanoscale circuits. Noual et al. [22] proposed a plasmonic multiplexer utilizing Y-bent MIM waveguides. However, this device needs at least $1.5 \mu \mathrm{m}$ of the distance between the cavities making it unsuitable for a compact device. Besides, the device was only designed for adding/dropping two operating wavelengths. Another plasmonic demultiplexer based on the metallic grating in threedimensional free space has been proposed [23] but its size seems to be unsuitable for the integration and miniaturization of devices due to the periodic array and 3D conformation. To promote the miniaturization of plasmonic devices, Hua Lu et al. [24] introduced a plasmonic triple wavelength demultiplexer, some others suggested wavelength demultiplexers based on nanocavities or coupled resonators [25]-[28]. However, investigations of such devices either suggested wavelengths not popular in WDM communication systems or the transmission efficiency is still relatively low.

In this paper, we designed a nanoplasmonic wavelength filter for three wavelength bands of $1310 \mathrm{~nm}, 1430 \mathrm{~nm}$ and $1550 \mathrm{~nm}$ which is based on the channel dropping structures in MIM waveguide using isolated Fabry-Perot (FP) rectangular resonators and a double stub waveguide. The eigen-mode expansion (EME) propagation simulation method [29] based on the highly-stable modal transmission line theory [30] has demonstrated the efficient transmission and relatively low loss of the drop waveguide when introducing the double nano-stub waveguide. We demonstrated that this nanoscale plasmonic triple-wavelength filter and 3-dB wavelength splitter has efficiently performed adding/dropping/splitting functions in applications of wavelength division multiplexing devices at the telecom regime such as optical access networks and optical computing systems.

\section{Model AND DESIGN PRINCIPLE}

The conceptual diagram of the proposed three-wavelength band filter is shown in Fig. 1. The device consists of $\mathrm{a} \mathrm{SiO}_{2}$ bus waveguide, a symmetric double nano-stub in the vertical 


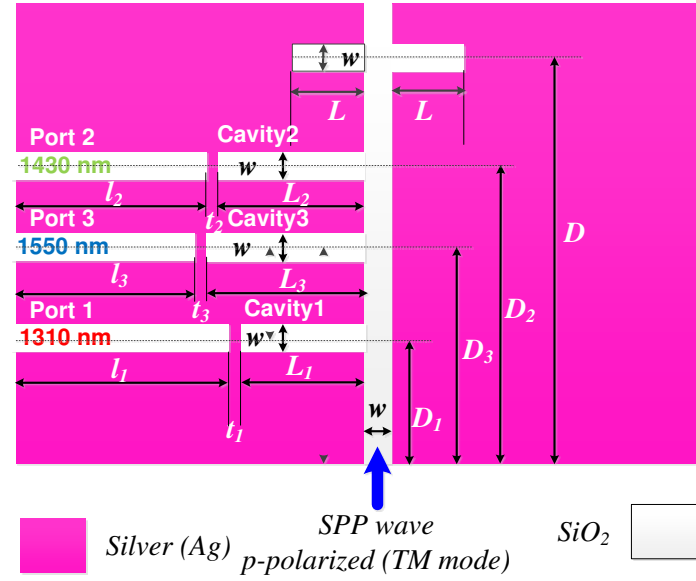

Fig.1. Conceptual schematic of a nanoplasmonic three-wavelength band filter based on MIM waveguide structure. All waveguides have a width of $w$. The double nano-stub has a length of $L$ for each aim. Three cavities named as Cavity1, Cavity2, Cavity3, and three output ports named as Port1, Port2, and Port3.

direction of the propagation waves and three $\mathrm{SiO}_{2}$ rectangular nanotube waveguides in the horizontal direction that are designed to selectively couple with each wavelength band for realizing the functionality of a drop channel. The purpose of the symmetric double stub is to make the possibility of wavelength band blocking for three bands of $1310 \mathrm{~nm}, 1430 \mathrm{~nm}$, and 1550 $\mathrm{nm}$. Working principle of the stub waveguide based on the principle of the temporal coupled mode theory. Whereas, plasmonic rectangular nanotube waveguides play the role of nanoscale isolated Fabry-Perot cavities, also following the principle of temporal coupled-mode theory [31],[32], to selectively couple wavelength bands depending on the appropriate cavity length. In this design, we labeled three nanocavities as Cavity1, Cavity2, Cavity3. These three cavities correspond to Port1, Port2, Port3 for the dropping channels of $1310 \mathrm{~nm}, 1430 \mathrm{~nm}$, and $1550 \mathrm{~nm}$ as seen in Fig. 1, respectively. The proposed structure based on MIM waveguides in which the insulating material in metallic slits and cavities is silica $\left(\mathrm{SiO}_{2}\right)$. Metallic layer in this device is silver, whose relative permittivity could be calculated by using the Drude model [19],[33] as follows:

$$
\varepsilon_{m}=\varepsilon_{\infty}-\frac{\omega_{p}^{2}}{\omega(\omega+i \gamma)}
$$

where $\varepsilon_{\infty}$ is the dielectric constant at the infinite frequency of silver; $\omega_{p}$ is the bulk plasma frequency; $\omega$ and $\gamma$ are the angular frequency of incident light in the vacuum and the electron oscillation damping constant, respectively. These parameters have following values $\varepsilon_{\infty}=3.7, \omega_{p}=9.1 \mathrm{eV}$, and $\gamma=0.018 \mathrm{eV}$ [34], [35]. Silver is selected in this device due to its very small imaginary part in the wavelength ranges of the telecom windows, thus, losses caused by the skin depth absorption are low enabling a long-range propagation of the SPP waves into the device. The refractive index of the fused silica glass material is described by the Sellmeier equation [36]

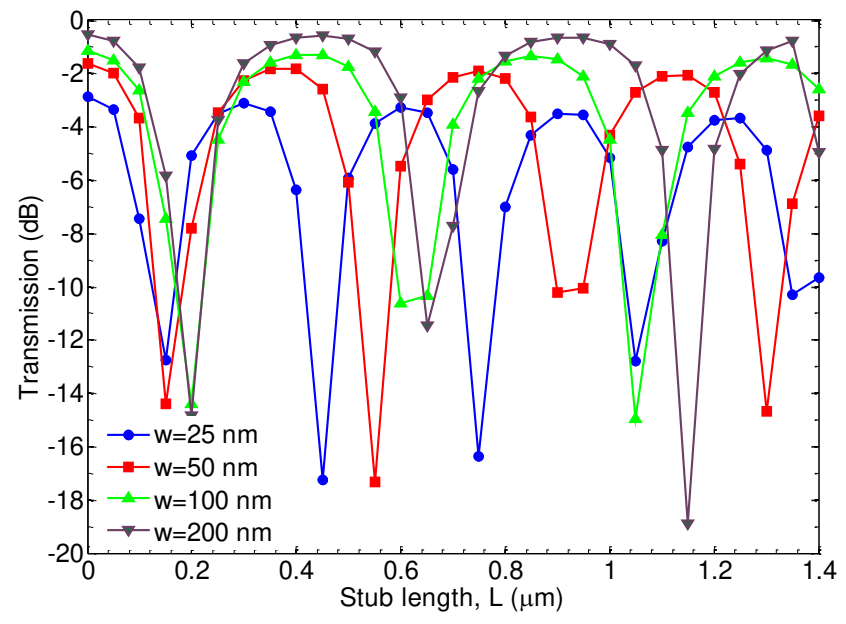

Fig.2. Transmission properties of the double stub waveguide solved by numerical simulation EME for different width of the stub at the wavelength of $1550 \mathrm{~nm}$.

in which its value in the wavelength range of the telecom regime varies in a short-range from 1.44 to 1.46 at room temperature of $300^{\circ} \mathrm{K}$ [37]. In this design, we make use of silica as an insulator instead of air because its high refractive index allows the MIM waveguide to confine lightwave in a nanoscale size easier than air.

As we all know that, following the surface plasmon polariton theory, the $s$-polarized wave (TE-polarized wave) cannot exist in the mechanism of the SPP mode. Whereas, only the $p$ polarized wave (TM-polarized wave) can exist and be guided in the mechanism of the SPP mode. Therefore, in our simulation study, the light of the TM-polarized wave is lead from the input port and propagates to three desired output ports for wavelength bands of $1310 \mathrm{~nm}, 1430 \mathrm{~nm}$, and $1550 \mathrm{~nm}$.

Transmission characteristics of the stub nano waveguide are analyzed by applying the temporal coupled-mode theory combining the plasmon waves passing through and returning from the stub with the phase matching. In that case, the transmission expressions of the symmetric single stub $\left(T_{s}\right)$ and double stub $\left(T_{d}\right)$ waveguides can be expressed as [38]:

$$
\begin{gathered}
T_{s}=\frac{1}{1+\tan ^{2}\left(\frac{2 \pi L}{\lambda_{g p}}\right)}, \\
T_{d}=\frac{4}{4+\tan ^{2}\left(\frac{2 \pi L}{\lambda_{g p}}\right)},
\end{gathered}
$$

here $\lambda_{g p}$ is the wavelength of the propagation plasmon in the MIM waveguide; $L$ is the length of the single stub structure.

Optical characterizations were carried out using EME simulation with the grid sizes in a two-dimensional space as $\Delta x$ $=\Delta z=5 \mathrm{~nm}$ applied for the total area of the proposed device. In this design, we choose all waveguides that have the same width, $w$. Fig. 2 shows the transmission curves of the single stub 


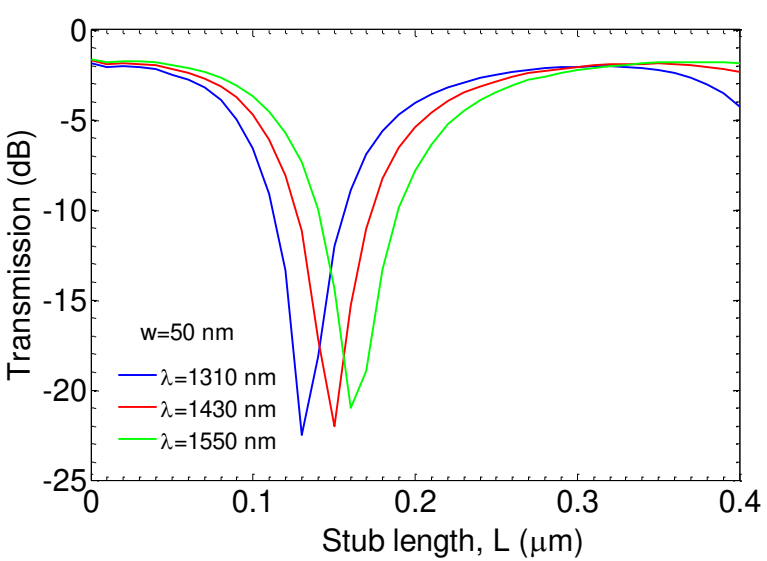

(a)

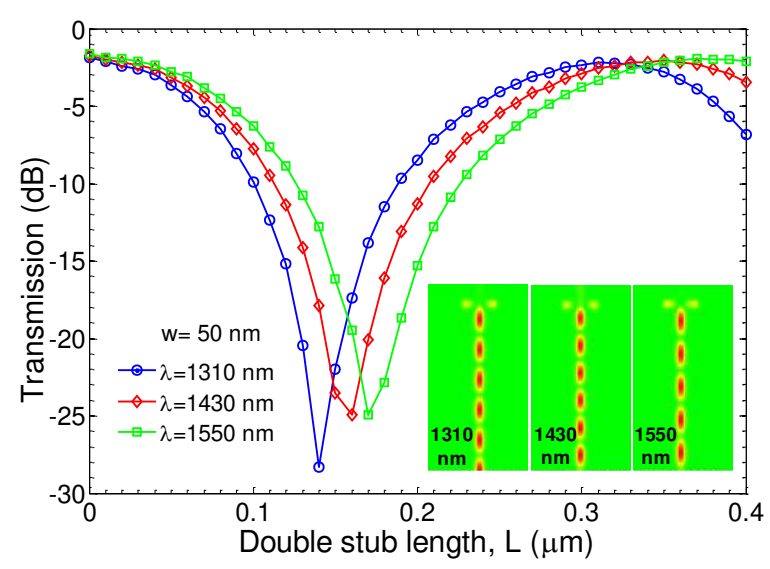

(b)

Fig. 3. Transmission properties of the stub waveguide via numerical simulation EME for three wavelength bands at the width $w=50 \mathrm{~nm}$ as a function of the stub length: (a) single stub, and (b) double stub. Insets in (b) are the electromagnetic field distributions for three wavelength bands.

waveguide at the output port as the functions of the single stub length $L$ for some values of the width $w$ at the operation wavelength of $1550 \mathrm{~nm}$. The waveguide widths for these studies are $w=25 \mathrm{~nm}, 50 \mathrm{~nm}, 100 \mathrm{~nm}$, and $200 \mathrm{~nm}$. Here, we placed the stub waveguides at a distance of $1.9 \mu \mathrm{m}$ from the input. As can be seen from Fig. 2, the wider stub width has higher efficiency. In addition, when the stub width increases the stub length for obtaining the highest resonance also increases. In order to realize the integrated circuit at the nanoscale in this design, we chose the width $w=50 \mathrm{~nm}$.

In Fig.3, transmission spectra characteristics are illustrated as a function of the stub length $L$ in the range from 0 to $0.4 \mu \mathrm{m}$ for three wavelengths of $1310 \mathrm{~nm}$ (blue), $1430 \mathrm{~nm}$ (red), and 1550 $\mathrm{nm}$ (green) for the single stub (a) and the double stub (b) at the width $w=50 \mathrm{~nm}$. Both sides of the double stub waveguide have the same length $L=160 \mathrm{~nm}$. The resonant peaks for these three wavelengths are $140 \mathrm{~nm}, 160 \mathrm{~nm}$, and $170 \mathrm{~nm}$ for both cases of single stub and double stub, respectively. Moreover, the maximum transmissions occur at a length of about $330 \mathrm{~nm}$ with a loss of $1.5 \mathrm{~dB}$. However, the full width at half maximum
(FWHM) of the resonant dips of the double stub is larger than that of the single stub for each corresponding wavelength. Furthermore, the depth of the resonant peaks corresponding to the minimum transmissions in the case of the double stub is significantly larger than that of the single stub. Simulation results are in good agreement with analytic characteristics depicted by Eq. (2) and Eq. (3). Insets in Fig. 3 (b) illustrate the distribution of electromagnetic fields by mean of eigen mode propagation method for three wavelength bands. It is seen that the double stub plays the role of a three bands holder, which helps enhance the efficiency of the dropping wavelengths in nano-cavities.

In this section, we will design the dropping channels by using nanotubes, which operate like FP cavities for selectively coupling at proper wavelength bands. First, we assume that the TM-polarized wave is injected into the device from the input bus waveguide with the incident power as $P_{I}$. We denote $O_{1}$, $\mathrm{O}_{2}$, and $\mathrm{O}_{3}$ for the drop ports with outgoing powers as $P_{\mathrm{O}_{1}}, P_{O_{2}}$ , and $P_{O_{3}}$, respectively. Transmission characteristics of the drop channels can be defined as $T_{d_{1}}=\frac{P_{O_{1}}}{P_{I}}, T_{d_{2}}=\frac{P_{O_{2}}}{P_{I}}$, and $T_{d_{3}}=\frac{P_{O_{3}}}{P_{I}}$.

For the plasmonic structure in our proposed MIM waveguides, the temporal evolution of amplitudes of incoming and outgoing waves are described by the interference between incident and reflected waves. These waves depend on the quality factors of the three nanotube cavities determined by the innate loss of propagating light over cavities. The accumulative phase between two reference planes satisfying the phase-matching condition is expressed by the following equation:

$$
\varphi=d \beta_{s p p}=d n_{e f f} k_{0}
$$

where $n_{\text {eff }}$ stands for the effective refractive index of the SPP mode propagating into the bus waveguide which depends on the wavelength $\lambda$ and the width $w$ of the bus waveguide; $k_{0}$ is the wave number of incident light in the vacuum; and $\beta_{s p p}$ represents the propagation constant of the SPP mode in the MIM waveguide. Eq (4) presents the phase-matching condition of the dispersion relation in the plasmonic waveguide which is governed by [19]:

$$
\varepsilon_{m} \sqrt{n_{e f f}^{2}-\varepsilon_{d}} \tanh \left(\frac{w_{t} k_{0} \sqrt{n_{e f f}^{2}-\varepsilon_{d}}}{2}\right)+\varepsilon_{d} \sqrt{n_{e f f}^{2}-\varepsilon_{m}}=0
$$

here $\varepsilon_{m}$ and $\varepsilon_{d}$ are the dielectric constants of the silver cladding and silica insulating layers. Three maximum transmissions of the dropping ports are expressed in the final forms owing to the mutual effects between cavities as follows [24]:

$$
\begin{gathered}
T_{d_{1}, \max }=\left|\frac{\left[1+r_{1}\right]^{2} /\left(2 Q_{d 1} Q_{1}\right)}{\left[1 / Q_{1}+1 /\left(2 Q_{d 1}\right)+\left(1+r_{1}\right) /\left(2 Q_{d 1}\right]^{2}\right.}\right| \\
T_{d_{2}, \max }=\left|\frac{\left[1+r_{2}\right]^{2} /\left(2 Q_{d 2} Q_{2}\right)}{\left[1 / Q_{2}+1 /\left(2 Q_{d 2}\right)+\left(1+r_{2}\right) /\left(2 Q_{d 2}\right]^{2}\right.}\right|
\end{gathered}
$$




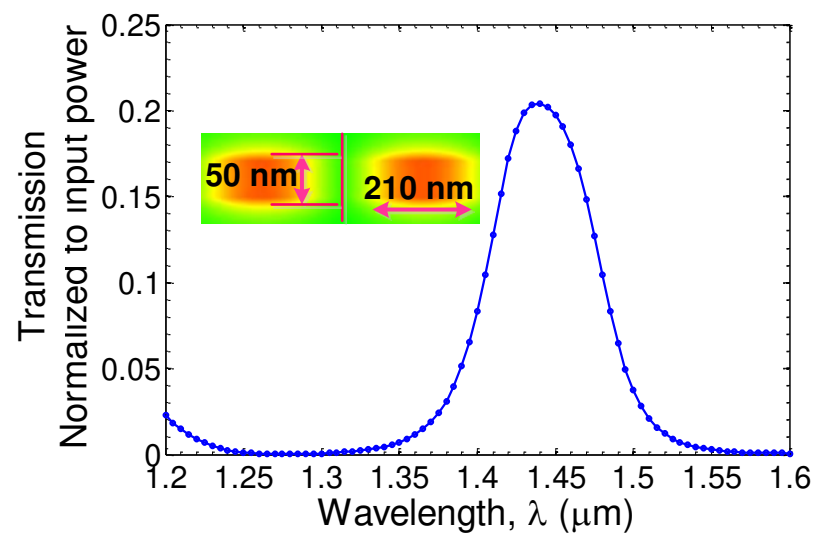

Fig. 4. EME simulation of the power transmission spectrum at the output Port2. The transmission is normalized to the input power. Inset: electromagnetic field in the nanoscale size of $50 \mathrm{~nm} \times 210 \mathrm{~nm}$ in the output Port2.

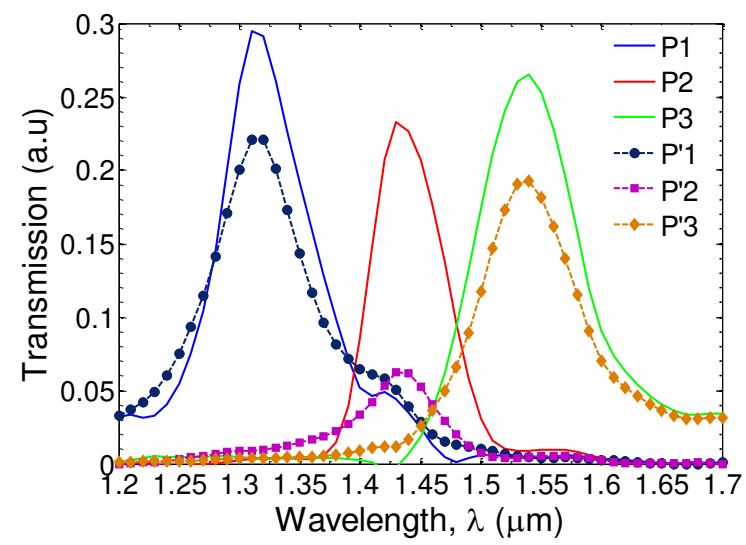

Fig. 5. EME simulation of normalized transmissions as a function of the wavelength for three output ports with (P1, P2, P3) and without (P'1, P'2, P'3) contribution of the double stub.

$$
T_{d_{3}, \max }=\left|\frac{\left[1+r_{3}\right]^{2} /\left(2 Q_{d 3} Q_{3}\right)}{\left[1 / Q_{3}+1 /\left(2 Q_{d 3}\right)+\left(1+r_{3}\right) /\left(2 Q_{d 3}\right]^{2}\right.}\right|
$$

where $Q_{1}, Q_{1}$, and $Q_{3}$ are the quality factors of Cavity1, Cavity2, and Cavity 3 , respectively; $Q_{d 1}, Q_{d 2}$, and $Q_{d 3}$ are decayed quality factors due to reducing of power into the dropping waveguides; $r_{1}=1 /\left[1+2 Q_{d 1} / Q_{1}\right], r_{2}=1 /\left[1+2 Q_{d 2} / Q_{2}\right]$, and $r_{3}=1 /\left[1+2 Q_{d 3} / Q_{3}\right]$ are the reduced quality factors from the bus waveguides due to the Ohmic absorption of the propagation waves.

Fig. 4 shows the normalized transmission amplitude of the magnetic field $\left|H_{y}\right|$ (TM-polarized SPP mode) that was simulated by utilizing the 2D-EME simulation method at the output Port 2 in the absence of the Cavity 1 and Cavity 3 as well as the double stub waveguides. The inset shows the electromagnetic field distribution of the TM mode $(\lambda=1430 \mathrm{~nm})$ in the nanoscale size of $50 \mathrm{~nm} \times 210 \mathrm{~nm}$ at the output Port2.

In order to separate three wavelengths bands into the desired output ports, we need to design Cavity1, Cavity2, and Cavity3 properly so that Cavity 1 is highly resonant with the wavelength of $1310 \mathrm{~nm}$ but is not resonant with the other wavelengths, and vice versa. In the same way, the Cavity 2 and Cavity 3 are designed for the resonant wavelengths of $1430 \mathrm{~nm}$ and 1550 $\mathrm{nm}$, respectively. Note that no analytical solution exists to describe the resonant effects of propagation waves in plasmonic waveguides. Thus, this paper will apply numerical simulation methods for investigation. Although both EME and FDTD (finite-difference time-domain) simulations are rigorous solutions for simulating plasmonic circuits that have been efficiently proved in many of the previous related works [35], [39]-[43], the FDTD method is time consuming on simulating and characterizing the circuit when compared to the EME method. To that end, in this paper, we utilize the EME method to simulate and optimize for design processes, nonetheless the obtained results are accurate.

In the simulation, we set the initial baffle thickness between rectangular cavities and the corresponding dropping waveguides as $t_{1}=t_{2}=t_{3}=t=10 \mathrm{~nm}$, the gap between cavities and the bus waveguide is also initially set the same value of $g=$ $0 \mathrm{~nm}$. We design selectively to realize highly efficient transmissions at the nanocavities via numerical simulation. The process is carried out by searching for the highest transmission efficiency at an accumulative phase condition of Cavity1 $\varphi_{1}=(2 m+1) \pi / 2$ for the wavelength of $1310 \mathrm{~nm}$ by changing both the distance $D_{1}$ and the length $L_{1}$ of Cavity1. Here $D_{1}$ denotes the distance from Cavity1 to the input port which was initially set as $455 \mathrm{~nm} . \mathrm{m}$ is an integer that we expect to be a minimum value, capably. Similar steps are also carefully performed in cases of Cavity2 and Cavity3. Finally, we found that the lengths of Cavity1, 2, and 3 are $400 \mathrm{~nm}, 640 \mathrm{~nm}$, and $575 \mathrm{~nm}$, respectively. The reference distances $D_{1}, D_{2}, D_{3}$ from the corresponding cavities numbers to the input port are found out as $455 \mathrm{~nm}, 1305 \mathrm{~nm}$, and $860 \mathrm{~nm}$ for obtaining the highest transmission efficiencies.

Fig. 5 presents the transmission efficiencies of the proposed drop filter at three desired output ports for three desired wavelength bands of $1310 \mathrm{~nm}, 1430 \mathrm{~nm}$, and $1550 \mathrm{~nm}$ by using numerical simulation in two cases, with and without the contribution of the double stub waveguide. Here, the normalized powers (to the input power) for the Port1, Port2, and Port3 are denoted as $\mathrm{P} 1, \mathrm{P} 2$, and $\mathrm{P} 3$ with the contribution of the double stub waveguide, and without this contribution as P'1, P'2, and P'3, respectively. Simulated results show that the transmission efficiencies of the SPP modes at the desired output ports are significantly enhanced for all three wavelength bands when introducing the double stub waveguides. Based on the working principle of the filter device, we can design a 3-dB three-wavelength band splitter by mirroring the output ports over the straight bus waveguide. The detailed characterization of these devices will be presented in the next section.

\section{DEVICE PERFORMANCE AND CHARACTERIZATION}

First, we use numerical simulations to evaluate the functions of the three-wavelength band filter and the three-wavelength band 3-dB splitter as well as characterize the optical 

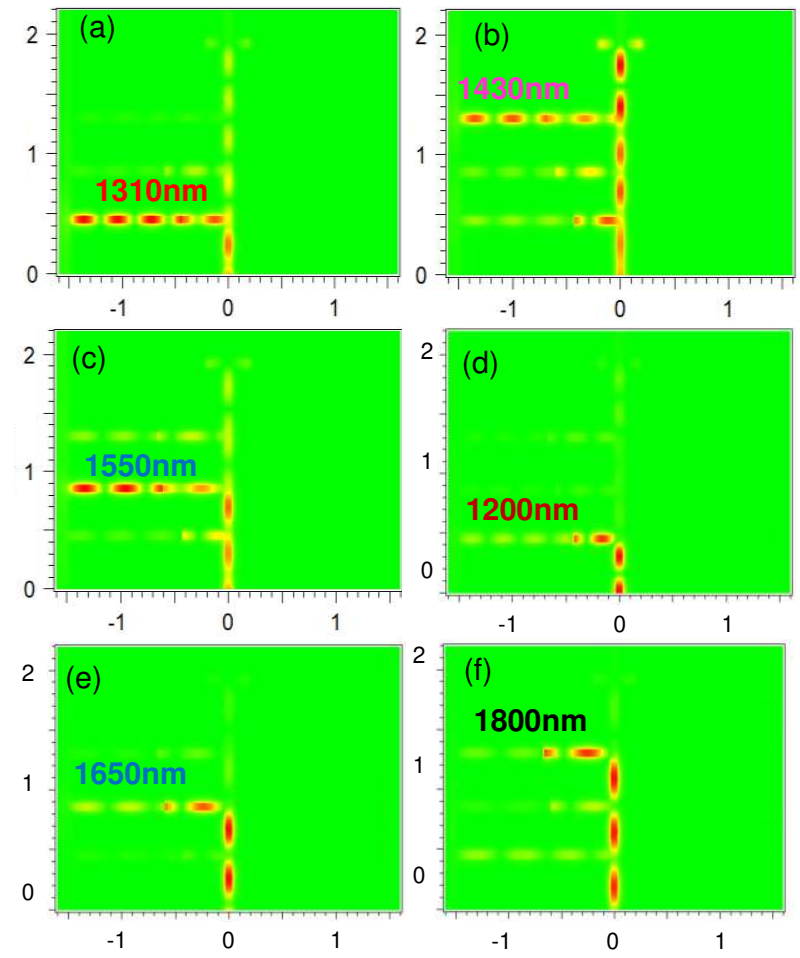

Fig. 6. Magnetic field amplitude distribution $\left|H_{y}\right|$ of plasmonic modes is simulated for three wavelength bands of $1310 \mathrm{~nm}$ (a), $1430 \mathrm{~nm}$ (b), and $1550 \mathrm{~nm}(\mathrm{c})$ for the proposed plasmonic wavelength filter; while (d), (e), (f) exhibit the confinement of light at Cavity1, 2, 3 for wavelengths of $1200 \mathrm{~nm}, 1650 \mathrm{~nm}$ and $1800 \mathrm{~nm}$, respectively. The axis units are in $\mu \mathrm{m}$.
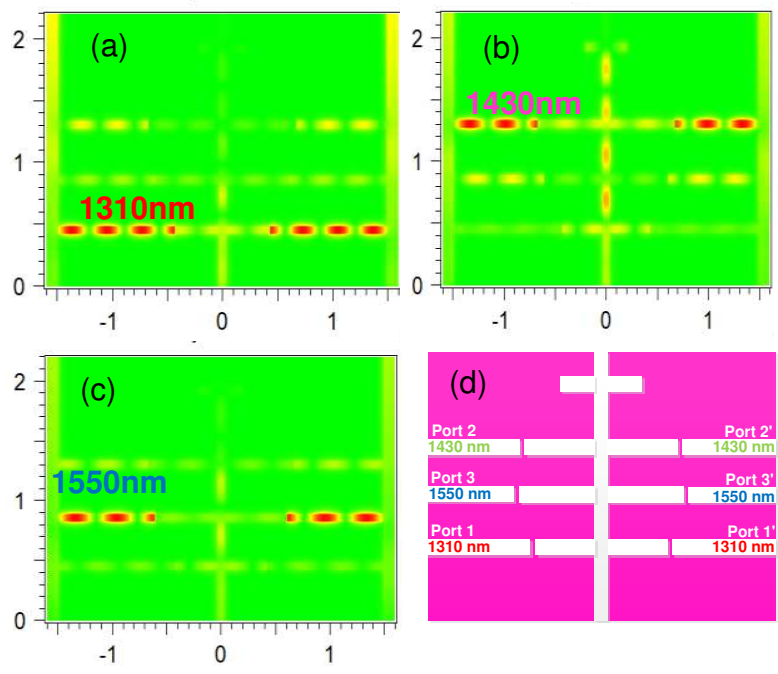

Fig. 7. Magnetic field amplitude distribution $\left|H_{y}\right|$ of plasmonic modes is simulated for three wavelength bands of $1310 \mathrm{~nm}$ (a), $1430 \mathrm{~nm}$ (b), $1550 \mathrm{~nm}$ (c) and structure diagram (d) for the proposed plasmonic 3$\mathrm{dB}$ wavelength splitter. The axis units are in $\mu \mathrm{m}$.

performances of the proposed device by mean of EME method. Fig. 6 (a), (b), (c) and Fig. 7 (a), (b), (c) show magnetic field distributions, $\left|H_{y}\right|$, of plasmonic modes at the individual wavelength bands for the wavelength filter and the wavelength splitter, respectively. The 2D-plots of the field distribution for the modes at three wavelength-bands clearly demonstrate functions of filter and 3dB-splitter of the proposed device with high efficiencies with negligible crosstalk between optical fields at each output ports. In addition, 2D-plots corresponding to Fig. 6 (d), (e), (f) also exhibit the confinement of light at Cavity1, 2, 3 for wavelengths of $1200 \mathrm{~nm}, 1650 \mathrm{~nm}$ and 1800 $\mathrm{nm}$, respectively.

Because the three-wavelength $3-\mathrm{dB}$ power splitter is designed by using a symmetrical structure to the design of three-wavelength filtering device, we only consider the characteristics of the proposed device in the role of the wavelength filter. We investigate the performance of this device in the wavelength domain by scanning wavelength from 1200 $\mathrm{nm}$ to $1700 \mathrm{~nm}$ via EME simulation. Fig. 8 illustrates the transmission properties of three output ports as functions wavelength. As can be seen, the power transmissions at three output ports obtain the highest resonant peaks at the wavelength bands of $1310 \mathrm{~nm}, 1430 \mathrm{~nm}$, and $1550 \mathrm{~nm}$ with the corresponding powers of $-5.37 \mathrm{~dB},-6.19 \mathrm{~dB}$, and $-5.68 \mathrm{~dB}$, respectively. Besides, a $3-\mathrm{dB}$ bandwidth $\left(\Delta \lambda_{F W H M}\right)$ of three bands obtained from the simulation exhibits the widths of 90 $\mathrm{nm}, 80 \mathrm{~nm}$, and $100 \mathrm{~nm}$ as presented by the blue, red, and green double arrows in Fig. 8, respectively. However, for assuring the difference level between the desired power and undesired powers, or in term of crosstalk, is not smaller than $10 \mathrm{~dB}$, the bandwidths for the output ports Port1, Port2, and Port3 in this case are as much as $90 \mathrm{~nm}, 40 \mathrm{~nm}, 100 \mathrm{~nm}$. The bandwidths of these calculated transmission spectra are relatively broad, and therefore, the proposed filter is suitable for application in broadband access optical networks. In order to make narrower bandwidth and increase the wavelength resolution for the wavelength selective filter in the application of wavelength division multiplexing (WDM) technique, we can modify the resonant properties of the isolated FP resonator to Fano-line shape resonator by adjusting the thickness, position of the silver baffles and the distance, and the length of FP resonators [44],[45].

To fabricate the MIM waveguide at the nanoscale size, high-resolution lithography techniques such as focused ionbeam (FIB) lithography, which based on heavier particles, can be used for patterning across a surface in order to create very small structures instead of using ultraviolet or X-ray photolithography techniques. Although the FIB technique has high accuracy, it still exists a finite tolerance because these heavier particles have more momentum for etching the patterns. Therefore, it is important to investigate the effect of geometrical fabrication tolerance regarding the width of cavities and the main bus waveguide for the performance of the proposed wavelength filter. Fig. 9 illustrates the variation of power transmissions at three output ports corresponding to the wavelength bands of $1310 \mathrm{~nm}$ (a), $1430 \mathrm{~nm}$ (b), $1550 \mathrm{~nm}$ (c), and total absorption and total reflection of the wavelength filter (d). With a tolerance of $\Delta w= \pm 2 \mathrm{~nm}$, the power transmissions are not smaller than $7 \mathrm{~dB}$, the crosstalk powers are below -15 $\mathrm{dB}$, the absorptions are not exceeded $1 \mathrm{~dB}$ and reflections are lower than $-10 \mathrm{~dB}$. 


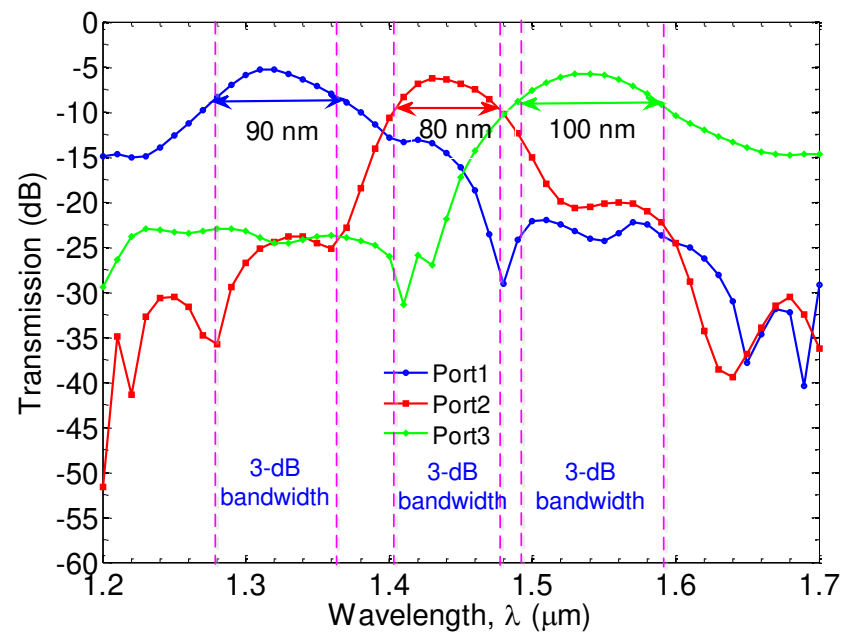

Fig. 8. Wavelength response for three desired output ports of the proposed plasmonic wavelength filter.
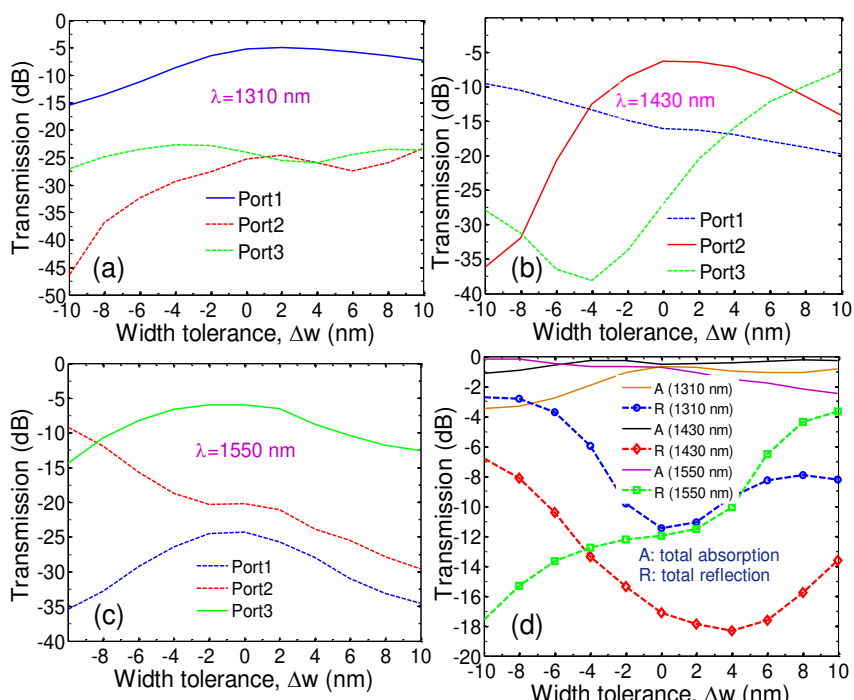

Fig. 9. Optical transmission characteristics as functions of width tolerance at three output ports: (a) Port1 at $1310 \mathrm{~nm}$, (b) Port2 at 1430 $\mathrm{nm}$, (c) Port3 at $1550 \mathrm{~nm}$, and (d) total absorption and total reflection at three wavelength bands for three output ports.

Finally, we study the effect of insulating materials on the characteristics of the proposed wavelength-selective filter. To do that we will investigate the performance of the device with the refractive index change, $\Delta n$, of different materials in comparison to that of the crystalline silica used in the previous section using the Sellmeier model [36]. Fig. 10 presents the power transmissions at output ports Port1, Port2, Port3, and output port Port0 of the main bus waveguide corresponding to wavelength bands of $1310 \mathrm{~nm}$ (a), $1430 \mathrm{~nm}$ (b), and $1550 \mathrm{~nm}$ (c). Fig. 10 (d) presents the total absorption and power transmission of individual wavelength bands. One can see a strong dependence of the optical characteristics at the output ports with the index difference of insulator materials. However, no clear behavior trend was observed rather the fluctuation. Both power transmission and absorption are the highest for silica material, about $-5 \mathrm{~dB}$ to $-6 \mathrm{~dB}$ for power transmission and $1 \mathrm{~dB}$ for absorption.
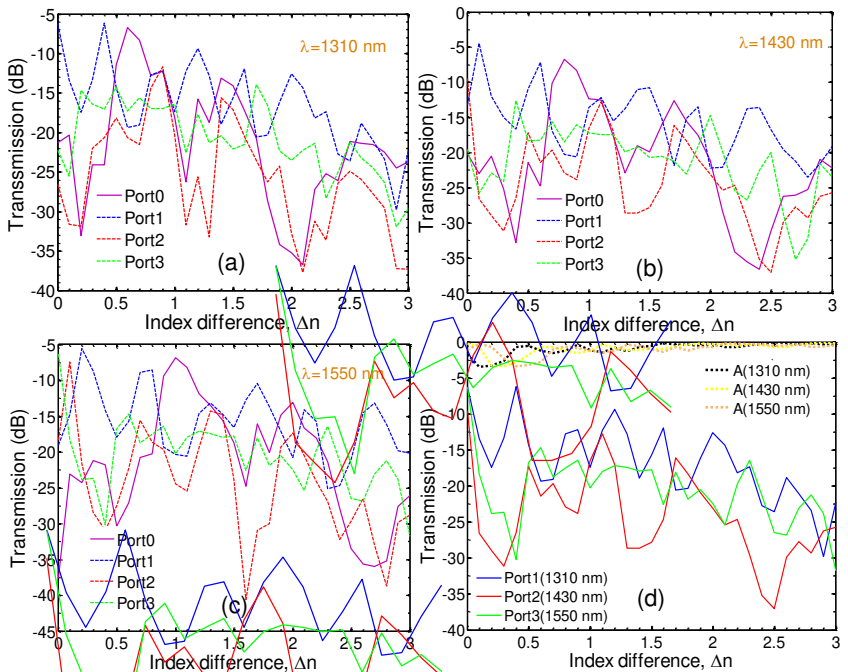

Fig. 10. Optical transmission characteristics as functions of index difference of insulating material at four output ports: (a) at $1310 \mathrm{~nm}$, (b) at $1430 \mathrm{~nm}$, (c) at $1550 \mathrm{~nm}$, and (d) total absorption and total power at three wavelength bands for three output port. $\Delta n=0$ is corresponding to silica.

\section{CONCLUSION}

In conclusion, a triple-wavelength filter using MIM waveguides with thin baffles and a double nanostubs was introduced as isolated FP resonators and wavelength band selector. Based on the strong coupling effect of various isolated FP resonators and enhancement of nanostub waveguide, a compact and highly efficient broadband wavelength filter and a broadband 3-dB wavelength splitter were demonstrated numerically via EME simulation. The proposed structure exhibits high transmission, low absorption, relative large tolerances, and relative broadband for applications in optical access networks at the telecom windows. In addition, the narrower bandwidth and the high wavelength resolution can be obtained via Fano-line shape resonance mechanism, which may provide a novel method for designing nanoscale and high resolution wavelength components in optical communications and computing, especially in wavelength division multiplexing and switching systems.

\section{ACKNOWLEDGEMENT}

This research is funded by Vietnam National Foundation for Science and Technology Development (NAFOSTED) under grant number 103.03-2017.61

\section{DECLARATIONS}

The authors declare no conflicts of interest.

\section{REFERENCES}

[1] W. L. Barnes, A. Dereux, and T. W. Ebbesen, "Surface plasmon subwavelength optics," Nature, vol. 424, pp. 824-830, 2003.

[2] D. K. Gramotnev and S. I. Bozhevolnyi, "Plasmonics beyond the diffraction limit," Nat. Photonics, vol. 4, no. 2, pp. 83-91, 2010.

[3] S. A. Maier, "Plasmonics: The Promise of Highly Integrated Optical Devices," IEEE J. Sel. Top. Quantum Electron., vol. 12, no. 6, pp. 16711677, 2006. 
[4] E. Ozbay, "Plasmonics : Merging Photonics and Electronics at Nanoscale Dimensions," Science (80-. )., vol. 311, pp. 189-193, 2012.

[5] C. H. Hsieh, K. P. Lin, and K. C. Leou, "Design of a Compact HighPerformance Electro-Optic Plasmonic Switch," IEEE Photonics Technol. Lett., vol. 27, no. 23, pp. 2473-2476, 2015.

[6] C. Haffner et al., "All-plasmonic Mach-Zehnder modulator enabling optical high-speed communication at the microscale," Nat. Photonics, vol. 9, no. 8, 2015.

[7] D. Dai and S. He, "A silicon-based hybrid plasmonic waveguide with a metal cap for a nano-scale light confinement.," Opt. Express, vol. 17, no. 19, pp. 16646-53, 2009.

[8] A. Melikyan et al., "High-speed plasmonic phase modulators," Nat. Photonics, vol. 8, no. 3, pp. 229-233, 2014.

[9] S. I. Bozhevolnyi, V. S. Volkov, E. Devaux, J. Laluet, and T. W. Ebbesen, "Channel plasmon subwavelength waveguide components including interferometers and ring resonators," Nature, vol. 440, pp. 508-511, 2006.

[10]L. Dong, H. Liu, S. Wang, S. Qu, and L. Wu, "Hybrid Tube-Triangle Plasmonic Waveguide for Ultradeep Subwavelength Confinement," $J$. Light. Technol., vol. 35, no. 11, pp. 2259-2265, 2017.

[11]C. Min and G. Veronis, "Absorption switches in metal-dielectric-metal plasmonic waveguides," Opt. Express, vol. 17, no. 13, p. 10757, 2009.

[12]H. Lu, X. Liu, L. Wang, Y. Gong, and D. Mao, "Ultrafast all-optical switching in nanoplasmonic waveguide with Kerr nonlinear resonator," Opt. Express, vol. 19, no. 4, p. 2910, 2011.

[13] X. Wu, J. Zhang, and Q. Gong, "Metal-insulator-metal nanorod arrays for subwavelength imaging.," Opt. Express, vol. 17, no. 4, pp. 2818-2825, 2009.

[14] S. H. Kwon, "Deep subwavelength-scale metal-insulator-metal plasmonic disk cavities for refractive index sensors," IEEE Photonics J., vol. 5, no. 1, 2013.

[15] Y.-C. Chang, C.-M. Wang, M. N. Abbas, M.-H. Shih, and D. P. Tsai, "Tshaped plasmonic array as a narrow-band thermal emitter or biosensor," Opt. Express, vol. 17, no. 16, p. 13526, 2009.

[16] J. Guo, "Plasmon-induced transparency in metal-insulator-metal waveguide side-coupled with multiple cavities," Appl. Opt., vol. 53, no. 8, p. 1604,2014

[17]M. Islam, D. R. Chowdhury, A. Ahmad, and G. Kumar, "Terahertz Plasmonic Waveguide Based Thin Film Sensor," J. Light. Technol., vol. 35, no. 23, pp. 5215-5221, 2017.

[18] J. H. Zhu, Q. J. Wang, P. Shum, and X. G. Huang, "A Nanoplasmonic High-Pass Wavelength Filter Based on a Metal-Insulator-Metal Circuitous Waveguide," vol. 10, no. 6, pp. 1357-1361, 2011.

[19] X.-S. Lin and X. G. Huang, "Tooth-shaped plasmonic waveguide filters with nanometric sizes," Opt. Lett., vol. 33, no. 23, pp. 2874-2876, 2008.

[20] Y. Liu, J. Yan, and G. Han, "The transmission characteristic of metaldielectric-metal slot waveguide-based nanodisk cavity with gain medium," IEEE Photonics J., vol. 7, no. 2, 2015.

[21] J. Xiao et al., "A CMOS-compatible hybrid plasmonic slot waveguide with enhanced field confinement," IEEE Electron Device Lett., vol. 37, no. 4 pp. 456-458, 2016.

[22] A. Noual, A. Akjouj, Y. Pennec, J.-N. Gillet, and B. Djafari-Rouhani, "Modeling of two-dimensional nanoscale Y-bent plasmonic waveguides with cavities for demultiplexing of the telecommunication wavelengths," New J. Phys., vol. 11, no. 103020, pp. 1-19, 2009.

[23] M. S. Kumar, X. Piao, S. Koo, S. Yu, and N. Park, "Out of plane mode conversion and manipulation of Surface Plasmon Polariton Waves," Opt. Express, vol. 18, no. 9, pp. 8800-8805, 2010.

[24]H. Lu, X. Liu, Y. Gong, D. Mao, and L. Wang, "Enhancement of transmission efficiency of nanoplasmonic wavelength demultiplexer based on channel drop filters and reflection nanocavities," Opt. Express, vol. 19, no. 14, pp. 12885-12890, 2011.

[25] X. M. Geng, T. J. Wang, D. Q. Yang, L. Y. He, and C. Wang, "Tunable Plasmonic Wavelength Demultiplexing Device Using Coupled Resonator
System," IEEE Photonics J., vol. 8, no. 3, 2016.

[26] I. Zand, A. Mahigir, T. Pakizeh, and M. S. Abrishamian, "Selective-mode optical nanofilters based on plasmonic complementary split-ring resonators," Opt. Express, vol. 20, no. 7, p. 7516, 2012.

[27]K. Wen et al., "Electromagnetically induced transparency-like transmission in a compact side-coupled t-shaped resonator," J. Light. Technol., vol. 32, no. 9, pp. 1701-1707, 2014.

[28] I. Zand, M. S. Abrishamian, and T. Pakizeh, "Nanoplasmonic loaded slot cavities for wavelength filtering and demultiplexing," IEEE J. Sel. Top. Quantum Electron., vol. 19, no. 3, pp. 1-5, 2013.

[29] A. Pannipitiya, I. D. Rukhlenko, M. Premaratne, H. T. Hattori, and G. P. Agrawal, "Improved transmission model for metal-dielectric-metal plasmonic waveguides with stub structure," Opt. Express, vol. 18, no. 6, p. 6191, 2010.

[30] S. E. Kocabas, G. Veronis, D. Miller, and S. Fan, "Transmission Line and Equivalent Circuit Models for Plasmonic Waveguide Components," IEEE J. Sel. Top. Quantum Electron., vol. 14, no. 6, pp. 1462-1472, 2008.

[31]H. Lu, X. Liu, D. Mao, L. Wang, and Y. Gong, "Tunable band-pass plasmonic waveguide filters with nanodisk resonators," Opt. Express, vol. 18 , no. 17, p. 17922, 2010.

[32]P. T. Kristensen, J. R. De Lasson, M. Heuck, N. Gregersen, and J. Mork, "On the Theory of Coupled Modes in Optical Cavity-Waveguide Structures," J. Light. Technol., vol. 35, no. 19, pp. 4247-4259, 2017.

[33]P. B. Johnson and R. W. Christy, "Optical constants of the noble metals," Phys. Rev. B, vol. 6, no. 12, pp. 4370-4379, 1972.

[34] J. Park, H. Kim, and B. Lee, "High order plasmonic Bragg reflection in the metal-insulator-metal waveguide Bragg grating," Opt. Express, vol. 16, no. 1, pp. 413-425, 2008.

[35]T. Søndergaard, J. Jung, S. I. Bozhevolnyi, and G. Della Valle, "Theoretical analysis of gold nano-strip gap plasmon resonators," New J. Phys., vol. 10, 2008.

[36]G. Ghosh, M. Endo, and T. Iwasaki, "Temperature-Dependent sellmeier Coefficients and Chromatic Dispersions for Some Optical fiber glasses," $J$. Light. Technol., vol. 12, no. 8, pp. 1338-1342, 1994.

[37]C. Z. Tan and J. Arndt, "Temperature dependence of refractive index of glassy $\mathrm{SiO} 2$ in the infrared wavelength range," J. Phys. Chem. Solids, vol. 61, no. 8, pp. 1315-1320, 2000.

[38] Y. Matsuzaki, T. Okamoto, M. Haraguchi, M. Fukui, and M. Nakagaki, "Characteristics of gap plasmon waveguide with stub structures," Opt. Express, vol. 16, no. 21, p. 16314, 2008.

[39] A. Dolatabady and N. Granpayeh, "All-optical logic gates in plasmonic metal-insulator-metal nanowaveguide with slot cavity resonator," $J$. Nanophotonics, vol. 11, no. 2, p. 026001, 2017.

[40]X. M. Geng, T. J. Wang, D. Q. Yang, L. Y. He, and C. Wang, "Tunable Plasmonic Wavelength Demultiplexing Device Using Coupled Resonator System," IEEE Photonics J., vol. 8, no. 3, pp. 1-8, 2016.

[41] Y. Yu, J. Si, Y. Ning, M. Sun, and X. Deng, "Plasmonic wavelength splitter based on a metal-insula tor-metal waveguide with a graded grating coupler," Opt. Lett., vol. 42, no. 2, p. 187, 2017.

[42]Y. J. He, "Investigation of LPG-SPR sensors using the finite element method and eigenmode expansion method," Opt. Express, vol. 21, no. 12, p. 13875, 2013.

[43]Y. N. Kulchin, O. B. Vitrik, and A. V. Dyshlyuk, "Analysis of surface plasmon resonance in bent single-mode waveguides with metal-coated cladding by eigenmode expansion method," Opt. Express, vol. 22, no. 18, p. 22196, 2014.

[44] J. Chen, Z. Li, J. Li, and Q. Gong, "Compact and high-resolution plasmonic wavelength demultiplexers based on Fano interference," Opt. Express, vol. 19, no. 10, p. 9976, 2011.

[45]Z. Chen, R. Hu, L. Cui, L. Yu, L. Wang, and J. Xiao, "Plasmonic wavelength demultiplexers based on tunable Fano resonance in coupledresonator systems," Opt. Commun., vol. 320, pp. 6-11, 2014. 


\section{Figures}

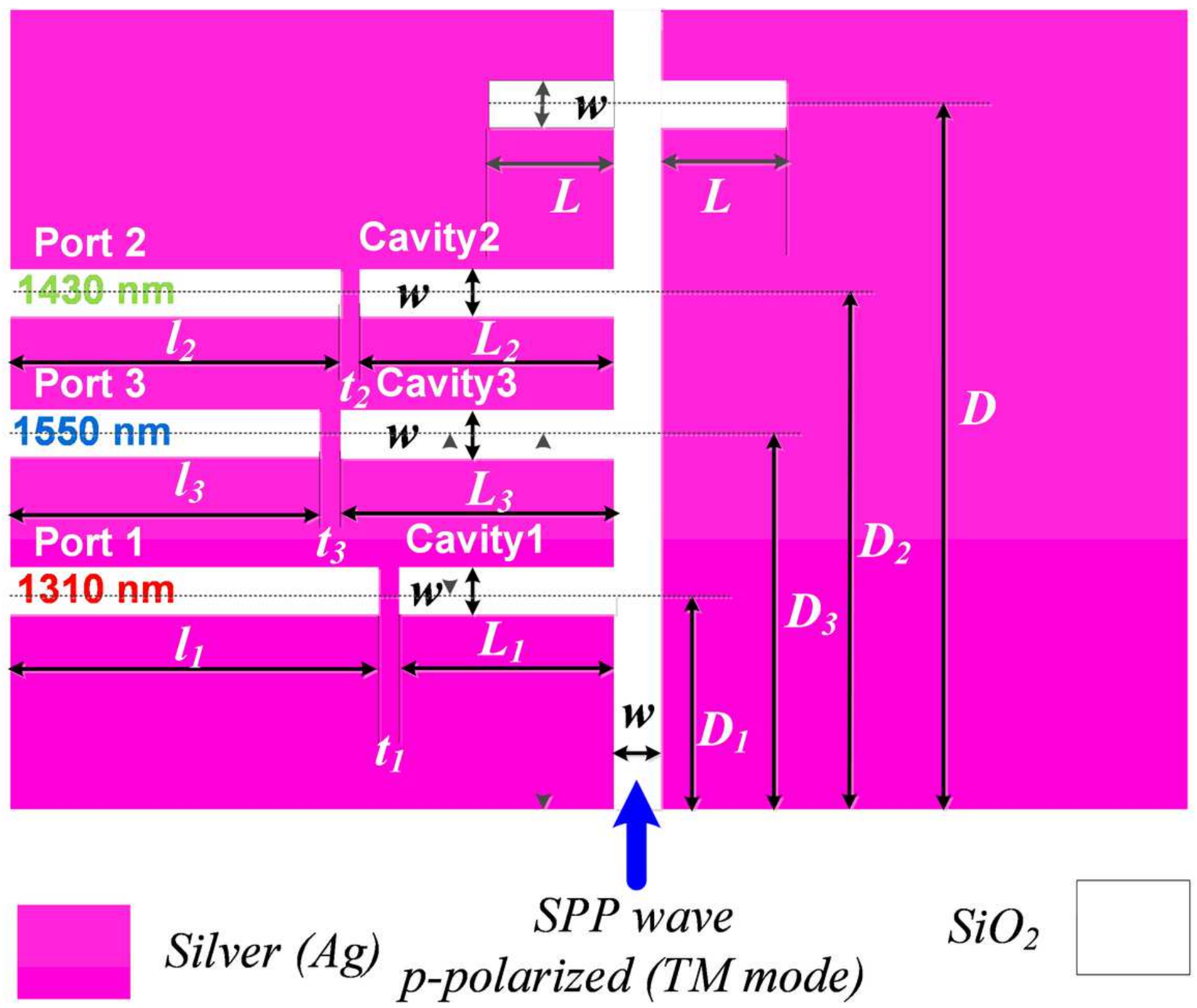

Figure 1

Conceptual schematic of a nanoplasmonic three-wavelength band filter based on MIM waveguide structure. All waveguides have a width of $w$. The double nano-stub has a length of $L$ for each aim. Three cavities named as Cavity1, Cavity2, Cavity3, and three output ports named as Port1, Port2, and Port3. 


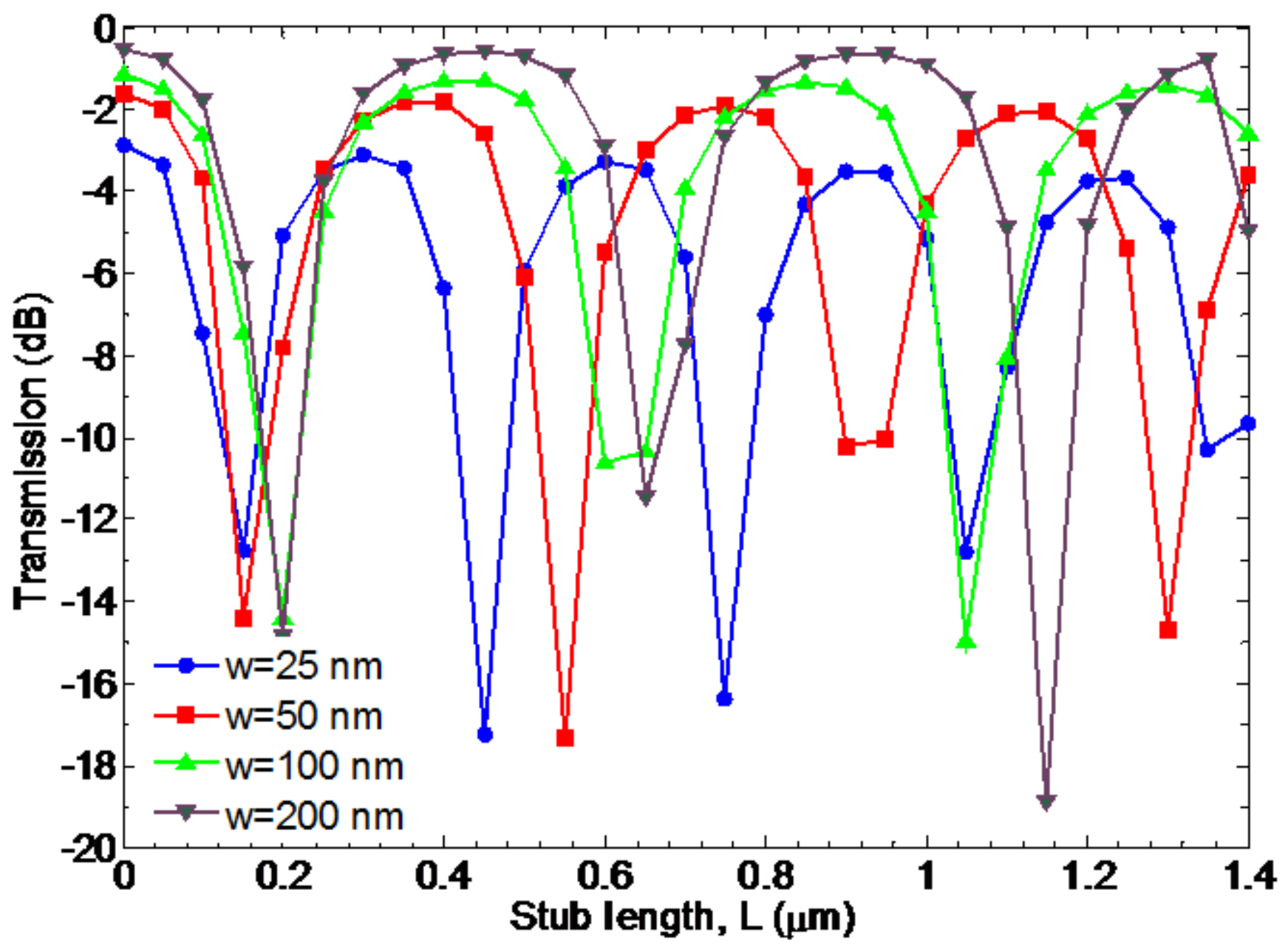

Figure 2

Transmission properties of the double stub waveguide solved by numerical simulation EME for different width of the stub at the wavelength of $1550 \mathrm{~nm}$.
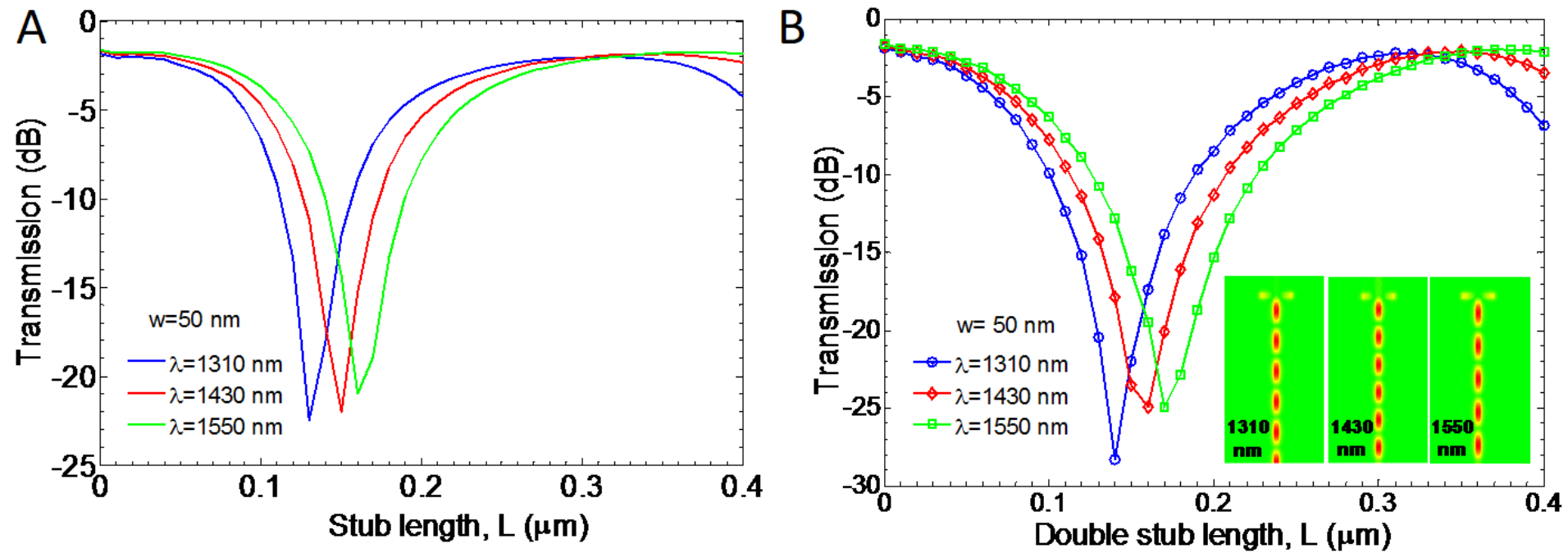

Figure 3 
Transmission properties of the stub waveguide via numerical simulation EME for three wavelength bands at the width $w=50 \mathrm{~nm}$ as a function of the stub length: (a) single stub, and (b) double stub. Insets in (b) are the electromagnetic field distributions for three wavelength bands.

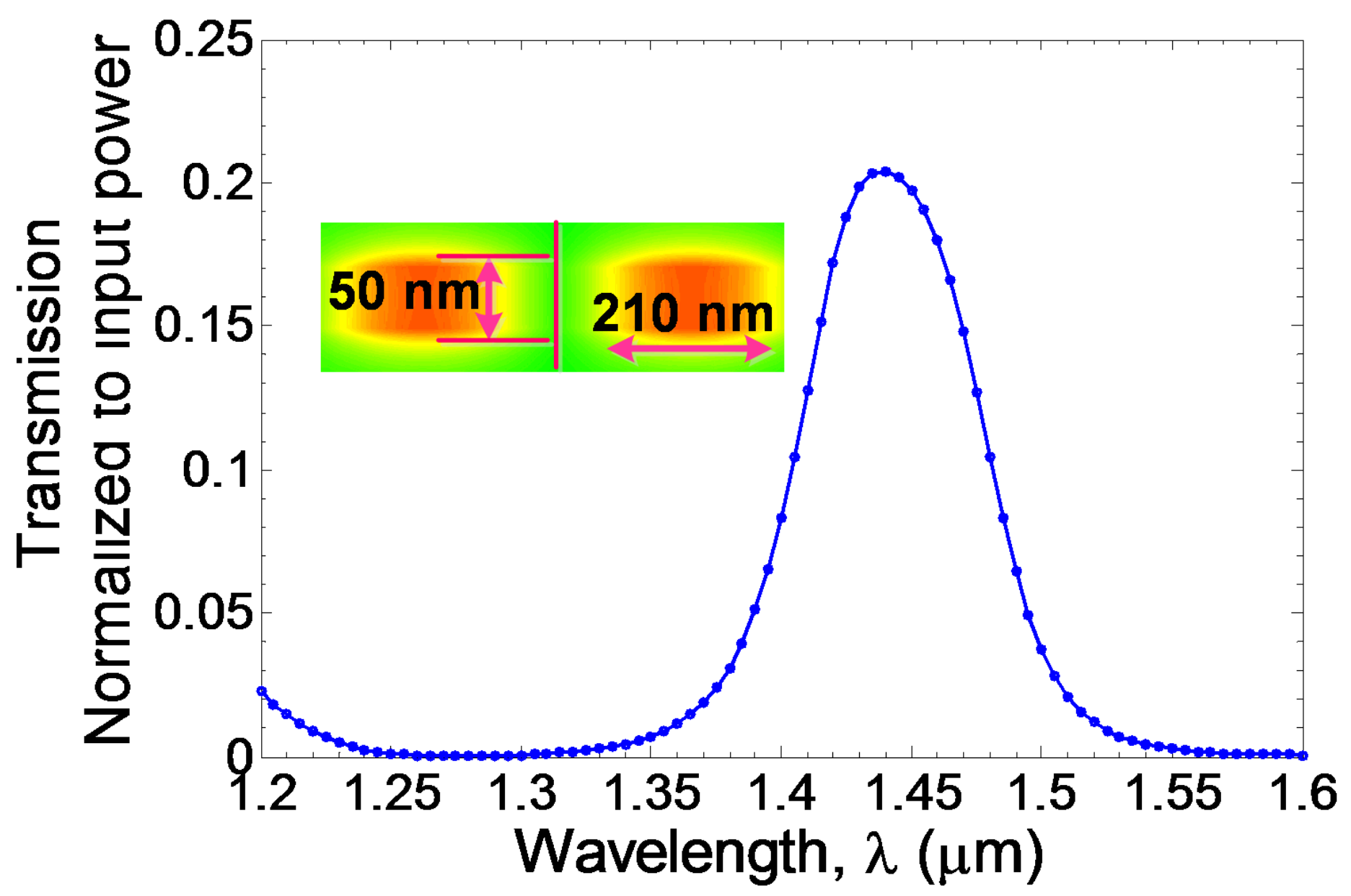

Figure 4

EME simulation of the power transmission spectrum at the output Port2. The transmission is normalized to the input power. Inset: electromagnetic field in the nanoscale size of $50 \mathrm{~nm} \times 210 \mathrm{~nm}$ in the output Port2. 


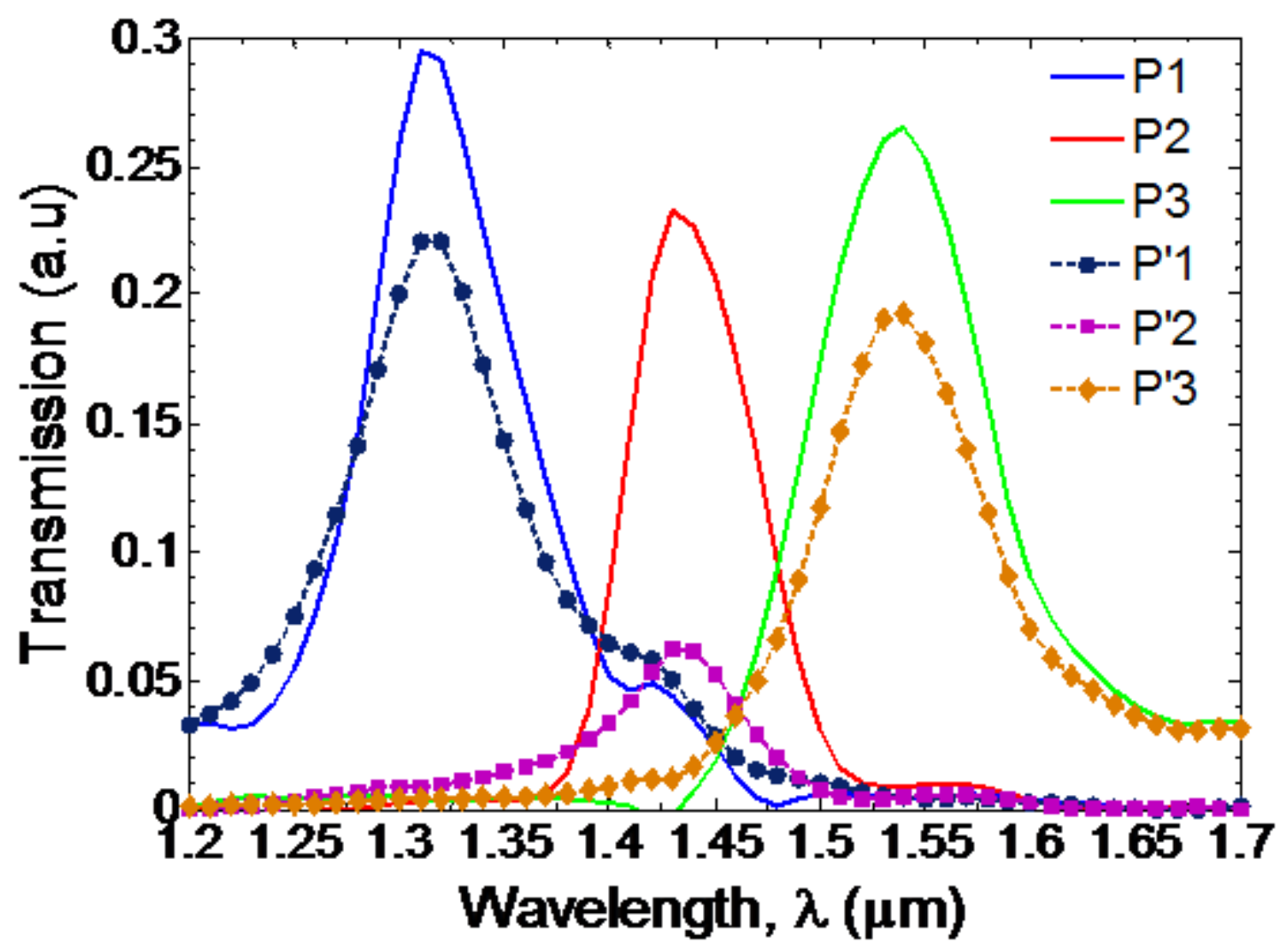

Figure 5

EME simulation of normalized transmissions as a function of the wavelength for three output ports with $(\mathrm{P} 1, \mathrm{P} 2, \mathrm{P} 3)$ and without $\left(\mathrm{P}^{\prime} 1, \mathrm{P}^{\prime} 2, \mathrm{P}^{\prime} 3\right)$ contribution of the double stub. 

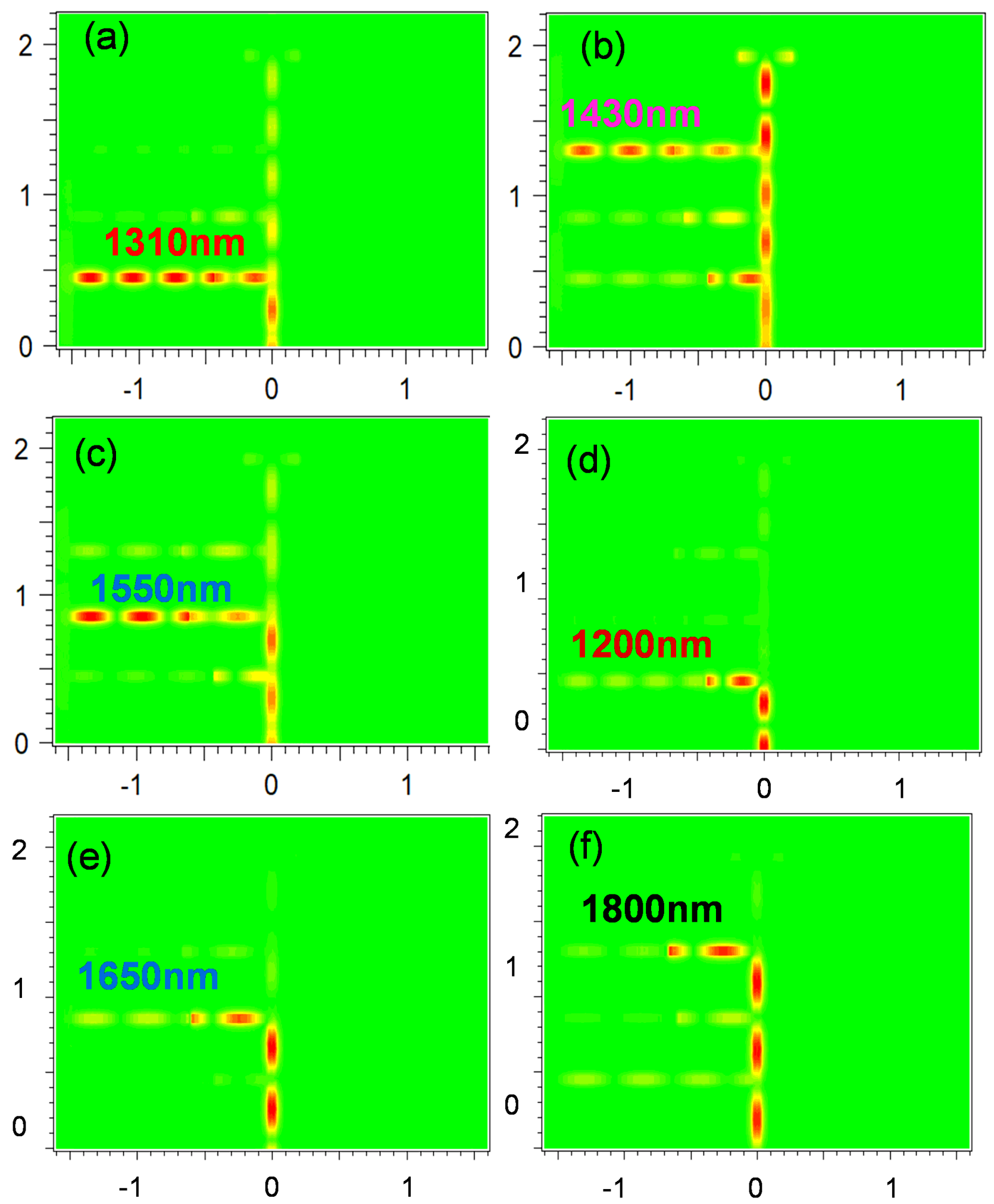

\section{Figure 6}

Magnetic field amplitude distribution $|\mathrm{Hy}|$ of plasmonic modes is simulated for three wavelength bands of $1310 \mathrm{~nm}(\mathrm{a}), 1430 \mathrm{~nm}$ (b), and $1550 \mathrm{~nm}$ (c) for the proposed plasmonic wavelength filter; while (d), (e), (f) exhibit the confinement of light at Cavity 1, 2, 3 for wavelengths of $1200 \mathrm{~nm}, 1650 \mathrm{~nm}$ and $1800 \mathrm{~nm}$, respectively. The axis units are in $\mu \mathrm{m}$. 

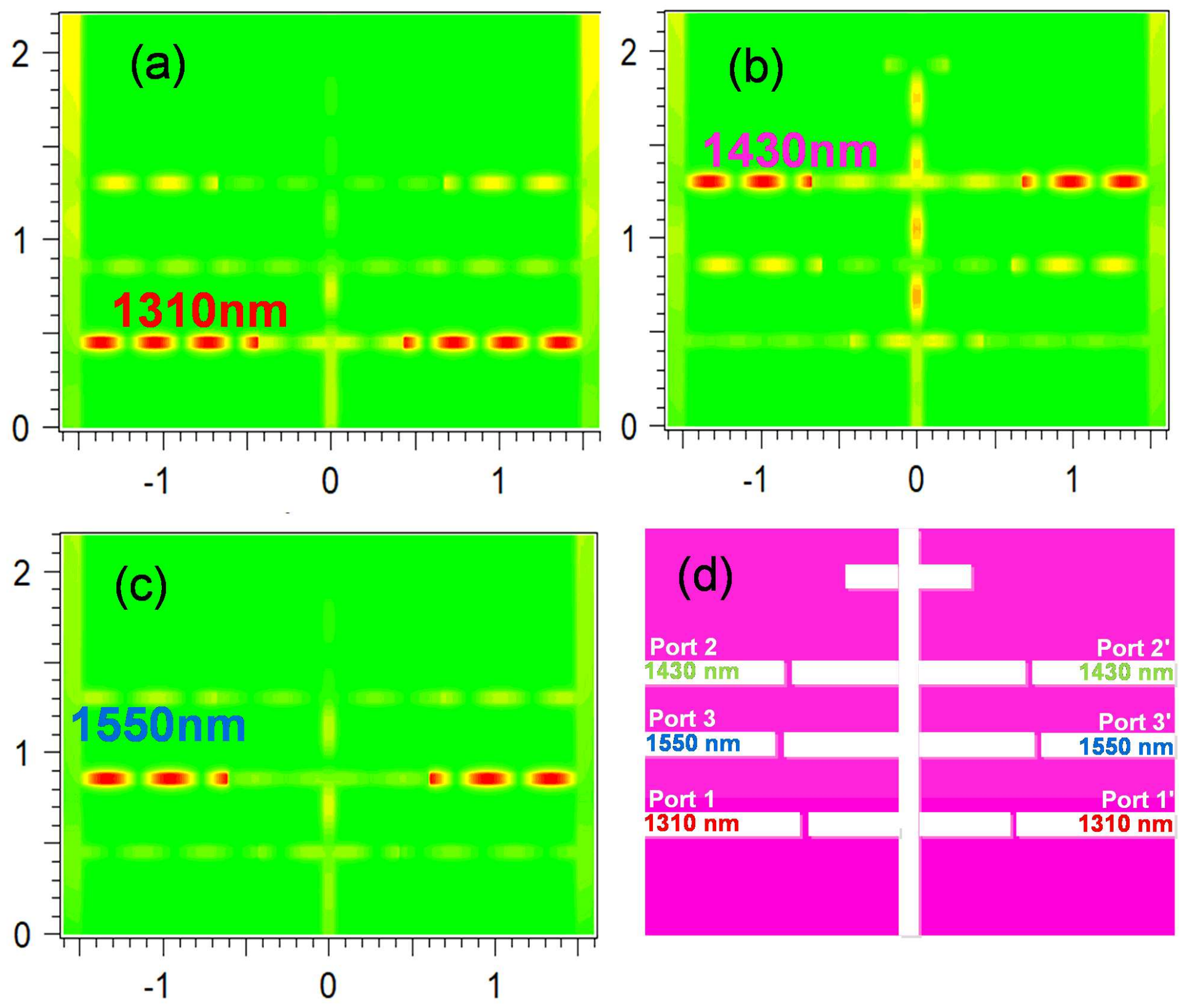

\section{Figure 7}

Magnetic field amplitude distribution $|\mathrm{Hy}|$ of plasmonic modes is simulated for three wavelength bands of $1310 \mathrm{~nm}$ (a), $1430 \mathrm{~nm}$ (b), $1550 \mathrm{~nm}$ (c) and structure diagram (d) for the proposed plasmonic 3-dB wavelength splitter. The axis units are in $\mu \mathrm{m}$. 


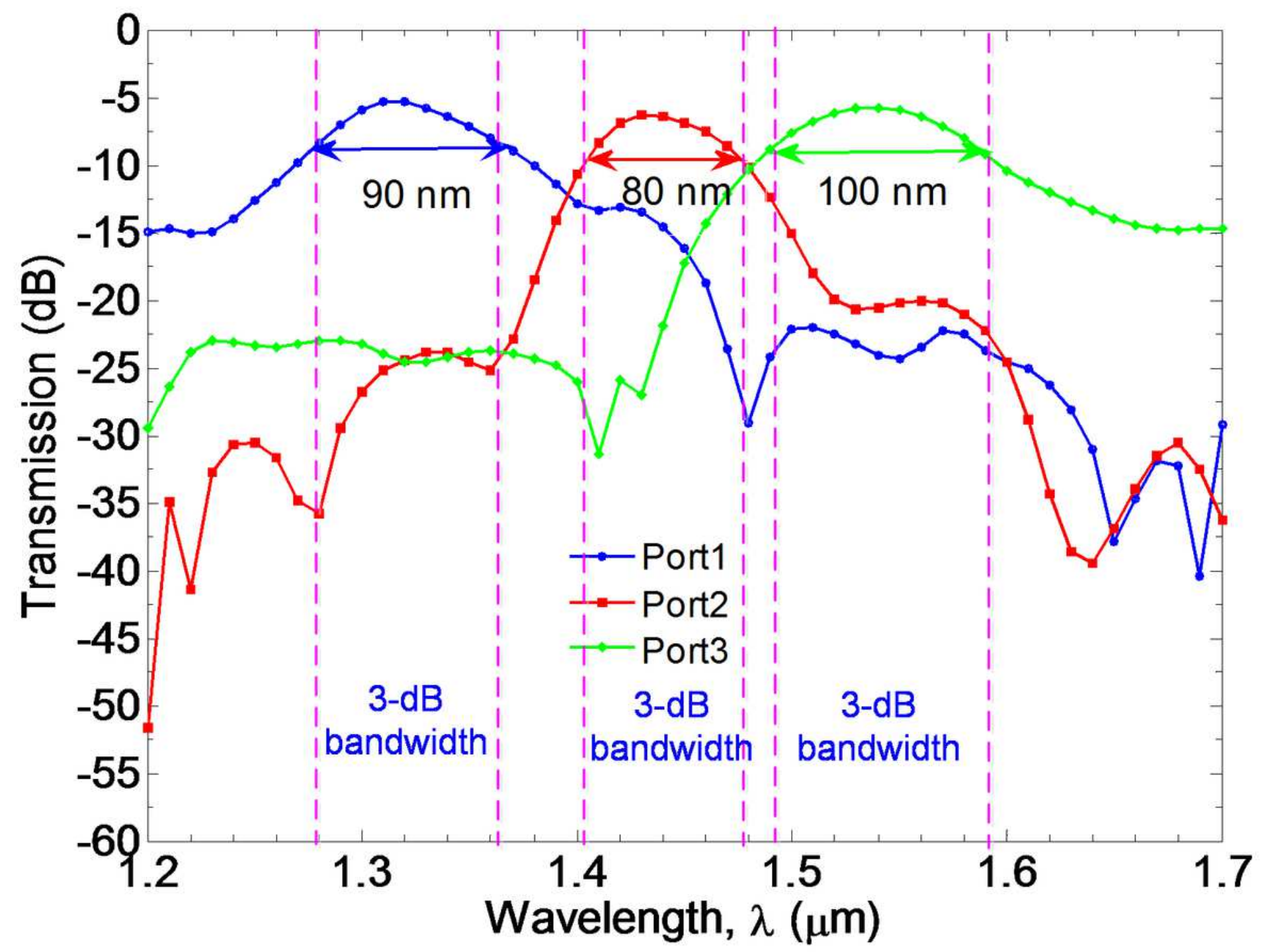

Figure 8

Wavelength response for three desired output ports of the proposed plasmonic wavelength filter. 

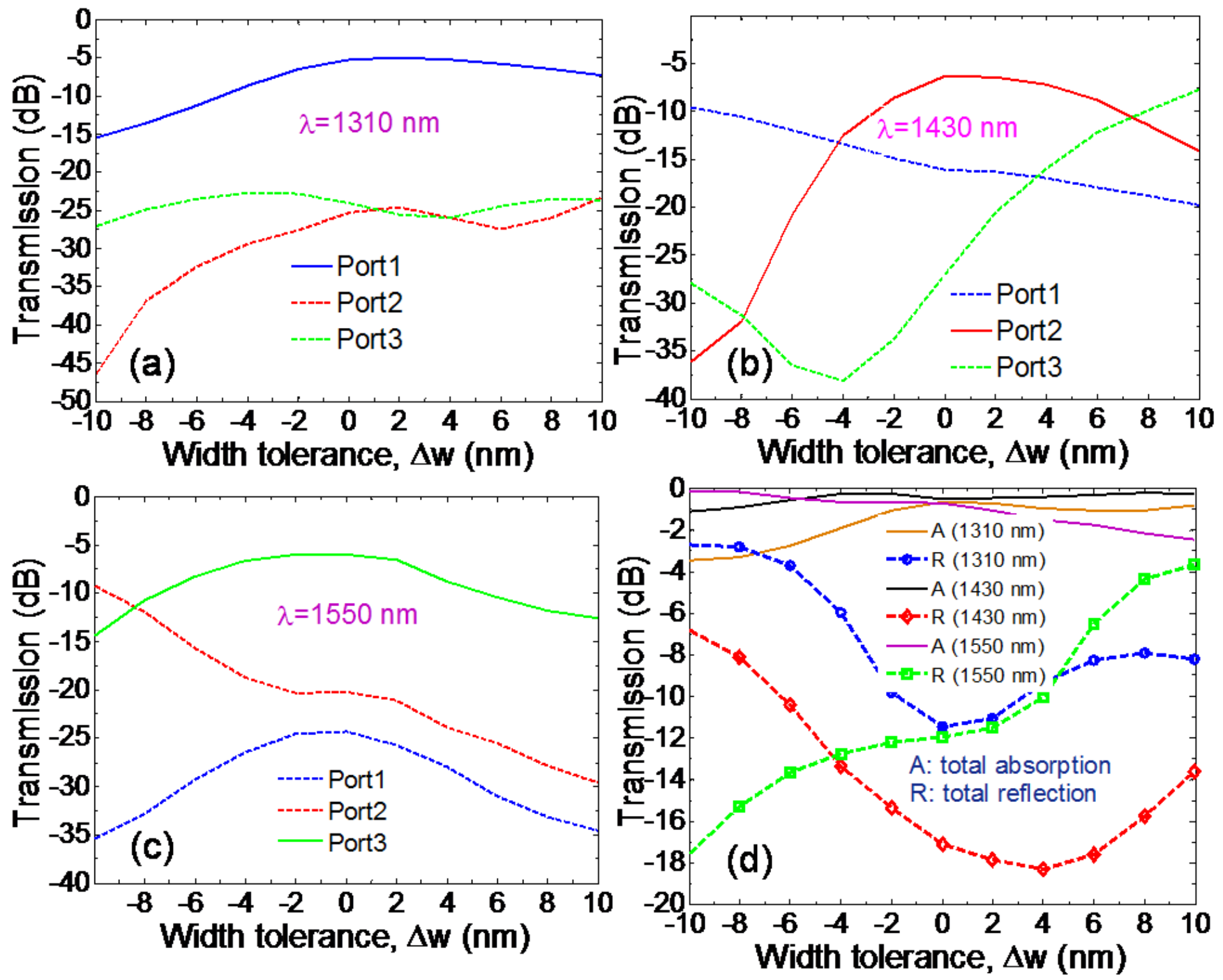

Figure 9

Optical transmission characteristics as functions of width tolerance at three output ports: (a) Port1 at $1310 \mathrm{~nm}$, (b) Port2 at $1430 \mathrm{~nm}$, (c) Port3 at $1550 \mathrm{~nm}$, and (d) total absorption and total reflection at three wavelength bands for three output ports. 

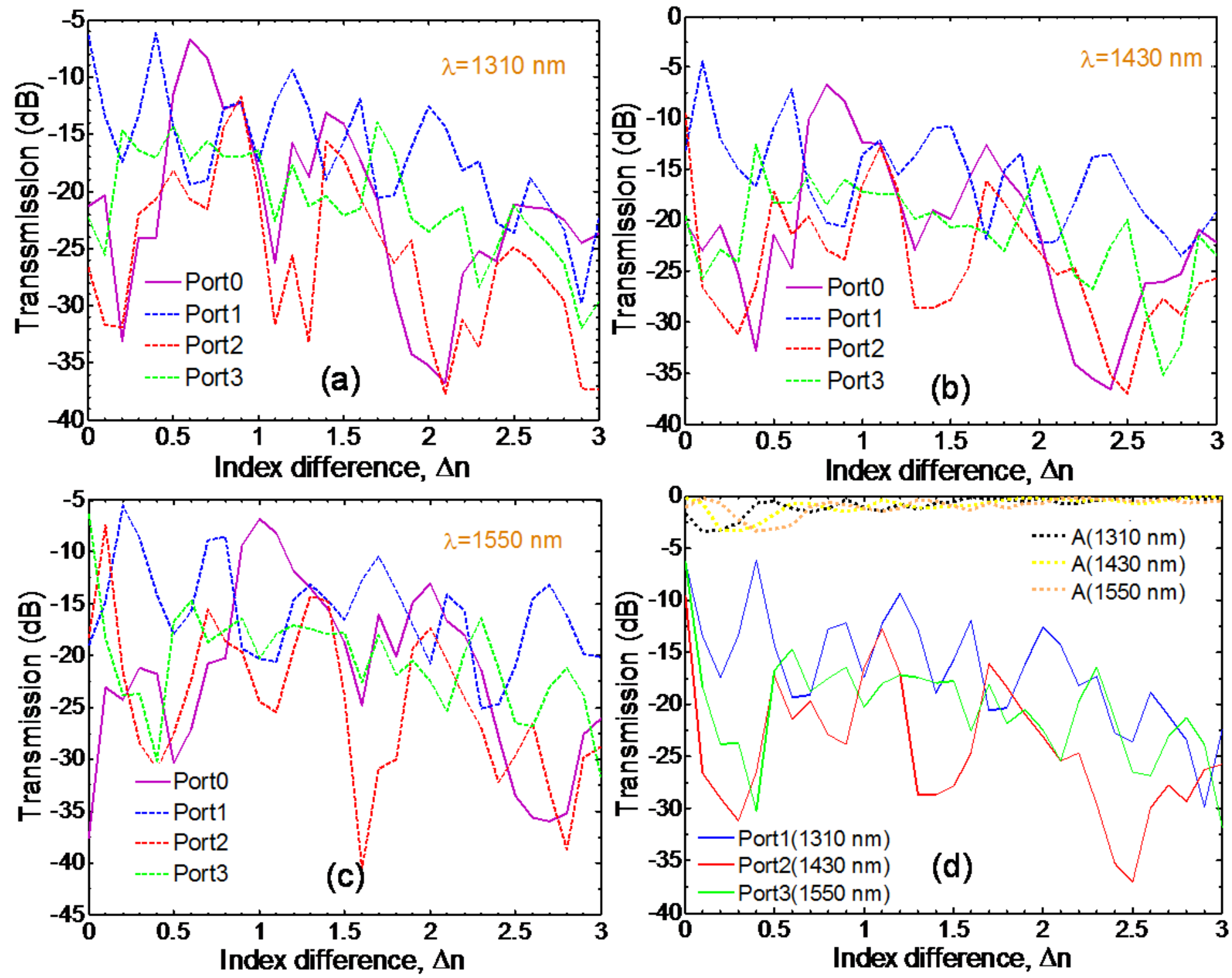

Figure 10

Optical transmission characteristics as functions of index difference of insulating material at four output ports: (a) at $1310 \mathrm{~nm}$, (b) at $1430 \mathrm{~nm}$, (c) at $1550 \mathrm{~nm}$, and (d) total absorption and total power at three wavelength bands for three output port. $\Delta \mathrm{n}=0$ is corresponding to silica. 